\title{
Euclidean vs. Graph Metric
}

\author{
Itai Benjamini
}

16.07 .12

\section{Introduction}

The theory of sparse graph limits concerns itself with versions of local convergence and global convergence, see e.g. [44]. Informally, in local convergence we look at a large neighborhood around a random uniformly chosen vertex in a graph and in global convergence we observe the whole graph from afar. In this note rather than surveying the general theory we will consider some concrete examples and problems of global and local convergence, with a geometric viewpoint. We will discuss how well large graphs approximate continuous spaces such as the Euclidean space. Or how properties of Euclidean space such as scale invariance and rotational invariance can appear in large graphs.

The first sections consider approximating the Euclidean and Finsler metrics by graphs. We study the emergence of rotational, scale and conformal invariance in large graph metrics. We then move on to comment on random graph metrics. Starting with graphs obtained by perturbing the Euclidean metric, and then moving on to random graphs that are restricted to have a planar topology. In particular, we will study graphs generated by random subdivisions. Local and global graph limits will be woven into the whole discussion.

\section{Notions of distance between metric spaces}

Given a graph $G=(V, E)$, the graph distance between any two vertices is the length of the shortest path between them. A graph $G$ is called vertex transitive if for any $u, v \in V$ there exists a graph automorphism mapping $u$ to $v$.

In the note we will consider three notions of distances between metric spaces.

The first is that of quasi-isometry and slack-isometry between spaces, the second the GromovHausdorff distance which is suitable for comparison between bounded spaces and is therefore useful for studying scaling limits, and the third is regarding a local statistical similarity between spaces.

See Burago and Ivanov [18] for background on metric spaces, including the first two notions and Lovász [44] for local limits. 
Definition 2.1. Two metric spaces $G$ and $H$ are said to be quasi-isometric if there exists a map $f: G \rightarrow H$ and two constants $1 \leq C<\infty$ and $0 \leq c<\infty$, such that

- $C^{-1} d_{H}(f(x), f(y))-c \leq d_{G}(x, y) \leq C d_{H}(f(x), f(y))+c$ for every $x, y \in G$,

- For every $y \in H$ there is an $x \in G$ so that $d_{H}(f(x), y)<c$.

Two metric spaces are said to be slack-isometric iff they are quasi-isometric with multiplicative constant equal to 1 . That is, if we can take $C=1$ in the definition.

For global convergence we use: the Gromov-Hausdorff distance between two metric spaces is obtained by taking the infimum over all the Hausdorff distances between isometric embeddings of the two spaces in a common metric space.

One way to look at a large finite graph is to look at a large neighborhood around a random uniformly chosen vertex. Often such neighborhood statistics capture quantities of interest and their asymptotics. Thus, one is led to take limits of such statistics and thereby define a probability measure on infinite rooted graphs, where the neighborhood of the root has the statistics that arise as the limit statistics of the finite graphs. Such a limit of a sequence of finite graphs is local limit. All such limit measures have a property known as unimodularity; it is not known whether all unimodular measures are limits of finite graphs. This fundamental question was asked in [2]. Those that are such limits are called sofic. Intuitively, a probability measure on rooted graphs is unimodular if its root is chosen "uniformly" from among all its vertices. This, of course, only makes sense for finite graphs. It is formalized for networks on infinite graphs by requiring a certain conservation property known as the Mass-Transport Principle, see [13] [2] [8].

For local limit we follow [13]: a limit of finite graphs $G_{n}$ is a random rooted infinite graph $(G, \rho)$ with the property that neighborhoods of $G_{n}$ around a random vertex converge in distribution to neighborhoods of $G$ around $\rho$.

Formally, let $(G, o)$ and $\left(G_{1}, o_{1}\right),\left(G_{2}, o_{2}\right), \ldots$ be random connected rooted locally finite graphs. We say that $(G, o)$ is the limit of $\left(G_{j}, o_{j}\right)$ as $j \rightarrow \infty$ if for every $r>0$ and for every finite rooted graph $\left(H, o^{\prime}\right)$, the probability that $\left(H, o^{\prime}\right)$ is isomorphic to a ball of radius $r$ in $G_{j}$ centered at $o_{j}$ converges to the probability that $\left(H, o^{\prime}\right)$ is isomorphic to a ball of radius $r$ in $G$ centered at $o$.

Given a (possibly random) graph we will consider the distribution on rooted graphs obtained by rooting at a random uniform vertex.

Exercise: what is the limit of $n$-level full binary trees?

Hint: it is not the infinite full binary tree.

In [13] it was shown that local limits of bounded degree graphs are a.s. recurrent for the simple random walk. A graph admits the square root separation property if for any vertex set $S$ in the graph, by removing not more than $|S|^{1 / 2}$ vertices from $S$, the connected components of $S$ has size at most $|S| / 2$. 
Question 2.2. Assume $\left\{G_{k}\right\}$ is a sequence of bounded degree graphs, all admitting the square root separation property. Is their limit recurrent?

Limits of graphs having $f(n)$-separation function, for some $f(n)$, suggests studying quantitative versions of Elek's hyperfinitness [44]. See [14] for more on separation.

Continuity of graph parameters with respect to local convergence is of current interest, here is one example.

Define $S A W(n)$ as the uniform measure on all the self-avoiding paths of length $n$ from a fixed root. By sub-multiplicativity $\mu=\lim |S A W(n)|^{1 / n}$ exists and is called the connective constant of the graph.

Conjecture 2.3. $\mu$ is continuous with respect to local convergence of infinite vertex transitive graphs.

We will also need the following notion,

Definition 2.4. Let $G=(V, E)$ be a finite graph. Define the Cheeger constant of $G$ to be

$$
h(G)=\inf _{0<|S|<\frac{|V|}{2}} \frac{|\partial S|}{|S|} .
$$

If $G$ is an infinite graph we set

$$
h(G)=\inf _{0<|S|<\infty} \frac{|\partial S|}{|S|} .
$$

An infinite graph $G$ with $h(G)>0$ is called non-amenable. Otherwise it is called amenable.

\section{Rotational invariance}

How well can the graph metric on bounded degree graphs approximate the metric of homogeneous manifolds equipped with some invariant length metric.

\subsection{Slack Euclidean?}

Recall that the scaling limit of the $\mathbb{Z}^{2}$ grid is the $l^{1}$ metric on the plane.

The following question was raised by Gady Kozma in a discussion with Oded Schramm and myself. Bruce Kleiner informed me after publication, that it was already asked by Erdős and Pach.

Question 3.1. Is there a bounded degree graph which is slack-isometric to the Euclidean plane?

The Pinwheel tiling, which is a non-periodic tiling defined by Charles Radin [50], is a graph quasi-isometric to the Euclidean plane where the multiplicative constant goes to 1 uniformly in the distance. 
By sampling a Poisson process in the Euclidean plane and drawing the corresponding Voronoi tiles we get the Poisson-Voronoi tessellation (see Wikipedia). The graph metric on the tiles is almost surely has an asymptotically Euclidean metric see e.g. Howard-Newman [29].

Question 3.2. What is the asymptotic shape of a ball in a Poisson-Voronoi tessellation where the underling space is the plane with an $l^{p}$ metric?

See the closely related [19].

\subsection{Near critical percolation}

Can the $l^{2}$ or other given Finsler metric "naturally" emerge as a limit of bounded degree graph metrics in the Gromov-Hausdorff distance?

Consider the natural embedding of the square grid in the plane.

Dilute the planar square grid by removing edges independently with probability $q<1 / 2$. Since $1 / 2$ is the critical percolation probability (Kesten [38]) almost surely there is a unique connected dense infinite subgrid left.

Condition on the origin to be in the infinite connected component and look at large balls rescaled to have diameter 1.

For any fixed $q$ the subadditive ergodic theorem was used in the context of first passage percolation to show that the rescaled large balls around the origin will a.s. converge in the Gromov-Hausdorff distance to a centrally symmetric convex body in the Euclidean plane.

Conjecture 3.3. As $q \rightarrow 1 / 2$ the limiting shape Gromov-Hausdorff converges to an Euclidean ball.

Seems hard, since metric properties do not follow from conformal geometry. Yet simple simulations seem convincing.

\section{Scale invariance}

\subsection{Rotational and scale invariant Euclidean structures}

Is there a distribution on tilings of the Euclidean plane which is rotation and translation invariant, mixing (that is, what is observed in far apart fixed Euclidean balls decorrelates with the distance between the balls), and stationary scale invariant (that is, there is a stationary matching or clustering of neighboring tiles resulting in a rescaled sample)? The Pinwheel tiling [50] is such. What if we further require spatial Markovity. That is given a tile you can not tell the tiling of the complement e.g. at which points of its boundary 3 tiles meet? Consider space filling Schramm's SLE(8) curve and remove from it an independent Poisson process in the plane, the curve is then cut into pieces of 
finite area. As suggested by Wendelin Werner, variants on this observation might provide the exotic tilings we are after.

Aldous [1] initiated a study of random road networks whose distributions are exactly invariant under Euclidean scaling. He introduced a natural axiomatization of a class of structures he called scale-invariant random spatial networks, whose primitives are routes between each pair of points in the plane and constructed a model, based on minimum-time routes in a binary hierarchy of roads with different speed limits, satisfying the axioms.

We mention briefly an open problem of remotely similar spirit. Can you foliate $\mathbb{R}^{d}$ with Brownian paths?

\section{One large scale control, symmetric graphs}

Let $\left(G_{n}\right)$ be an unbounded sequence of finite, connected, vertex transitive graphs such that $\left|G_{n}\right|=$ $o\left(\operatorname{diam}\left(G_{n}\right)^{d}\right)$ for some $d>0$. In [10] the following theorem is shown.

Theorem 5.1. After taking a subsequence and rescaling by the diameter, the sequence $\left(G_{n}\right)$ converges in the Gromov-Hausdorff distance to a torus of dimension $<d$, equipped with some invariant Finsler metric.

In particular, if the sequence admits a doubling property at a large yet sub diameter scale, then the limit will be a torus equipped with some invariant length metric. Otherwise it will not converge to a finite dimensional manifold. When the degrees are uniformly bounded the limiting metric is a polygonal Finsler metric.

The proof relies on a recent quantitative version of Gromov's theorem on groups with polynomial growth obtained by Breuillard, Green and Tao [17] and a scaling limit theorem for nilpotent groups by Pansu [48]. See also Gelander [32]. Establishing quantitative versions will have applications to random walks and percolation on vertex transitive graphs. For example in the spirit of Varopoulos' theorem that the only recurrent finitely generated groups have at most quadratic growth [52]:

Let $G$ be a finite, $d$-regular connected vertex transitive graph. View $G$ as an electrical network in which each edge is a one Ohm conductor.

Conjecture 5.2 (with Gady Kozma). For any two vertices

$$
\text { electric resistence }(v, u)<C_{d}+\frac{\operatorname{diam}^{2}(G) \log |G|}{|G|} .
$$

In addition for a sequence of vertex transitive graphs, if the diameter is $o\left(\left|G_{n}\right|\right)$ then the electric resistance between any two vertices is $o\left(\operatorname{diam}\left(G_{n}\right)\right)$. 
Since finite vertex transitive graphs, when they converge to a manifold, converge to a torus, it follows that the infimum, over all such, of the Gromov-Hausdorff distance to $S^{n}$ is attained. Which one is the closest?

Question 5.3. Is the skeleton of the truncated icosahedron (soccer ball) the closest to $S^{2}$ ?

"Proof": Otherwise we would have a different design for soccer balls. See also Géode (géométrie) in French Wikipedia.

\subsection{Expander at all scales?}

A sequence of graphs $\left\{G_{n}\right\}$ is of an expander if there is $h>0$, for all $n, h\left(G_{n}\right)>h$.

Question 5.4. Is there a family $\left\{G_{n}\right\}$ of finite d-regular graphs, $\left|G_{n}\right| \rightarrow \infty$, so that all the induced balls in all the $G_{n}$ 's are expanders?

That is, there is $h>0$, for all $r>0$ and any $v$ in any of the graphs $G_{n}$ 's the ball $B(v, r)$ is $h$ expander, expander with a uniform edge expansion constant $h$. Note e.g. that if $G_{n}$ is a sequence of expanders with girth growing to infinity, then if $r$ is smaller than the girth then the balls of radius $r$ are trees and thus they are not uniform expanders as $r$ grows.

We conjecture that there is no such family. For vertex transitive graphs a positive answer to the following conjecture regarding percolation on expanders will show that no such family exists. The proof will proceed by constructing a limiting nonamenable vertex transitive graph with a unique infinite cluster whenever percolation occurs, we omit the outline.

Question 5.5. Let $G$ be a bounded degree expander, further assume that there is a fixed vertex $v \in G$, so that after performing $p=1 / 2$ percolation on $G$,

$$
P_{1 / 2}(\text { the connected component of } v \text { has diameter }>\operatorname{diameter}(G) / 2)>1 / 2 \text {, }
$$

Is there a giant component w.h.p? $G$ is not assumed to be transitive.

The following two questions are regarding the rigidity of the global structure given local information.

Question 5.6. Given a fixed rooted ball $B(o, r)$, assume there is a finite graph such that all its $r$-balls are isomorphic to $B(o, r)$, e.g. $B(o, r)$ is a ball in a finite vertex transitive graph, what is the minimal diameter of a graph with all of its r-balls isomorphic to $B(o, r)$ ? Any bounds on this minimal diameter, assuming the degree of $o$ is d? Any example where it grows faster than linear in $r$, when $d$ is fixed? 
Note that some $r$-ball in the grandparent graph, or any infinite non-unimodular vertex transitive graphs, does not appear as a ball in a finite vertex transitive graph. As by [13] local limit of finite graphs is unimodular. When the rooted ball is a tree, this is the girth problem. One can consider a weaker version e.g. when we require only that most balls are isomorphic to $B(o, r)$. Not assuming a bound on the degree, consider the 3 -ball in the hypercube, is there a graph with a smaller diameter than the hypercube so that all its 3-balls are that of the hypercube?

Question 5.7 (with Romain Tessera). Let $X$ is the Euclidean or hyperbolic plane, together with a triangulation, whose triangles are at most of diameter $r$. Suppose for each pair of Euclidean (or hyperbolic) balls of radius $r, B_{1}, B_{2}$ centered on vertices of this triangulation, there is a Euclidean (or hyperbolic) isometry mapping $B_{1}$ to $B_{2}$ respecting the triangulation (in the obvious way).

Does it imply that the triangulation is periodic?

\subsubsection{Roughly transitive graphs}

A metric space $X$ is $(C, c)$-roughly transitive if for every pair of points $x, y \in X$ there is a $(C, c)$ quasi-isometry sending $x$ to $y$.

If $G_{n}$ is only roughly transitive and $\left|G_{n}\right|=o\left(\operatorname{diam}\left(G_{n}\right)^{1+\delta}\right)$ for $\delta>0$ sufficiently small, we are able to prove, this time by elementary means, that $\left(G_{n}\right)$ converges to a circle.

Question 5.8. Is there an infinite $(C, c)$-roughly transitive graph, with $C, c$ finite, which is not quasi-isometric to a homogeneous space?

Here a homogeneous space is a metric space with a transitive isometry group. The same question can be asked in the wider category of Coarse embeddings.

See [6] and references there for the study of quasi-isometry between random spaces.

\section{Packing}

Packing one graph in another space can be viewed as large scale-rough conformal geometry. Large scale conformal geometry is developed in a work by Pierre Pansu [49]. We present a sample.

Question 6.1. Which graphs can be realized as the nerve graph of a sphere packing in Euclidean d-dimensional space?

Here vertices correspond to spheres with disjoint interiors and edges to pairs of touching spheres.

The rich two dimensional theory started with Koebe, who proved that every planar graph admits a circle packing.

In higher dimensions, Thurston observed that packability implies an upper bound of order $|G|^{(d-1) / d}$ on the size of minimal separators, see e.g. [46]. There is an emerging theory with many 
still open directions. Local graph limits were useful in the proof of the last two theorems below. Denote by $T_{3}$ the 3 -regular tree.

Theorem 6.2 (with Oded Schramm). The grid $\mathbb{Z}^{4}, T_{3} \times \mathbb{Z}$ and lattices in hyperbolic 4 -space do not admit sphere packing in Euclidean $\mathbb{R}^{3}$.

Let $\left(G_{n}\right)$ be a sequence of finite, $(k>2)$-regular graphs with girth growing to infinity.

Theorem 6.3. For every $d$ there exists an $N(d)$ such that $G_{n}$ does not admit a regular sphere packing in Euclidean d-dimensional space, for any $n>N(d)$.

The following is an extension to higher dimension of a theorem of Bowditch [16] following a suggestion by Gromov.

Theorem 6.4. Let $G$ be an infinite locally finite connected graph which admits a regular packing in $\mathbb{R}^{d}$. Then we have the following alternative: either $G$ has a positive Cheeger constant, or there are arbitrarily large subsets $S$ of $G$ such that $|\partial S|<|S|^{\frac{d-1}{d}+o(1)}$.

By regularly we mean uniform upper bound on the ratio of the radii of neighboring spheres. The proof of the last two theorems in [7] uses sparse graphs limits: by [13] local limits of bounded degree finite planar graphs are a.s. recurrent for the simple random walk, in [7] the proof was adapted to show that local limit of finite graphs that are regularly packed in $\mathbb{R}^{d}$, are $d$-parabolic. Which is the key to the results above.

Question 6.5 (with Oded Schramm). Show that any packing of $\mathbb{Z}^{3}$ in $\mathbb{R}^{3}$ has at most one accumulation point in $\mathbb{R}^{3} \cup\{\infty\}$.

\section{Perturbing the Euclidean metric}

Some families of metric spaces are naturally parameterized by the reals. The critical spaces are usually more exotic. We will present a few examples. These spaces sometimes admit combinations of properties which are impossible in the vertex transitive world. We start with the classical model of first passage percolation for perspective.

\subsection{First passage percolation}

One natural way to randomly perturb the Euclidean planar metric is that of first passage percolation (FPP), see [39] and [33] for background. That is, consider the square grid lattice, denoted $\mathbb{Z}^{2}$, and to each edge assign an i.i.d. random positive length. There are other ways to randomly perturb the Euclidean metric and many features are not expected to be model dependent. Large balls converge after rescaling to a convex centrally symmetric shape. Richardson (1973) proved the first shape 
theorem, when the length has exponential distribution and the graph is the $\mathbb{Z}^{d}$ lattice. Simulations indicate that the limiting shape is not the Euclidean ball. Kesten (unpublished) showed that the shape is not the Euclidean ball in high enough dimension.

The boundary fluctuations are conjectured to have a Tracy-Widom distribution. The variance of the distance from the origin to $(n, 0)$ is conjectured to be of order $n^{2 / 3}$. So far only an upper bound of $\frac{n}{\log n}$ was established, see [11]. Optimal bounds on the length of efficient algorithms for finding the shortest path or to estimate its length are still unknown.

The structure of geodesic rays and two-sided infinite geodesics in first passage percolation is still far from being understood. Furstenberg asked in the 80's (attending a talk by Kesten) to show that almost surely there are no two sided infinite geodesics for natural FPP's, e.g. exponential length on edges.

Häggström and Pemantle introduced [26] competitions based on FPP, see [23] for a survey. Here is a related problem. Start two independent simple random walks on $\mathbb{Z}^{2}$ walking with the same clock, with the one additional condition, that the walkers are not allowed to step on vertices already visited by the other walk, and otherwise choose uniformly among allowed vertices. Show that almost surely, one walker will be trapped in a finite domain. Prove that this is not the case in higher dimensions.

\subsection{Pertubations, beyond first passage percolation}

We now describe several random metrics, the first two of which can be viewed as perturbations of the grid like FPP, but with slightly stronger perturbation "causing the underlying grid metric to almost disappear".

\subsubsection{LRP}

Start with the one dimensional finite grid $\mathbb{Z} / n \mathbb{Z}$ with the nearest neighbor edges, add additional edges to it as follows. Between, $i$ and $j$ add an edge with probability $\beta|i-j|^{-s}$, independently for any pair. The main problem in long range percolation is, how does the distance between 0 and $n / 2$ typically grows in this random graph?

The off critical cases: when $s>2$ the distance is of order $n$, for $1<s<2$ the distance is polylogn (see [15] for the exact result, background and history). For $s=1$ Coppersmith, Gamarnik and Sviridenko showed that the distance is $\frac{\log n}{\log \log n}$ and if $s<1$ the distance is uniformly bounded.

The critical case: when $s=2$ the distance is of the form $\theta\left(n^{f(\beta)}\right)$, where $f$ is strictly between 0 and 1 (Sly and Ding [24]). Continuity, monotonicity, or even a guess of $f$ are still open. We believe that there is a scaling limit for the $s=2$ long range percolation random graphs.

These natural random graphs admit a combination of properties which is impossible for vertex transitive graphs. E.g. when $1<s<2$ the mixing time of the simple random walk is a.s. $n^{s-1}$. That is, small diameter does not exclude small bottlenecks as in vertex transitive graphs [5]. 


\subsubsection{CCCP}

Examine bond percolation on $\mathbb{Z}^{d}$. Each edge is open with probability $p$ independently. Clusters are connected components of open edges. For any $d>1$, there is $0<p_{c}<1$, such that if $p<p_{c}$ all the clusters are finite a.s. and the diameter of the clusters has exponential tail. If $p>p_{c}$ there is a unique infinite cluster. While for the critical probability $p_{c}$ it is conjectured that there is no infinite cluster and that the diameter of clusters has polynomial tail. This is true in dimensions 2 and $d$ large.

The unique infinite cluster, for $p>p_{c}$ is a random perturbation of the grid. E.g. asymptotics of the heat kernel are the same, how can we get "interesting" critical geometry?

Conditioning on the critical percolation to have an infinite cluster results in a "thin" graph with infinitely many cut points.

Here is a suggestion: contract each cluster into a single vertex. The result is a random graph $G$ of high degree (each vertex $v \in G$ is a cluster $\mathcal{C}$ in $\mathbb{Z}^{d}$ and its degree is the number of closed edges coming out of $\mathcal{C}$ ). When the percolation is subcritical one expects to see a perturbation of the lattice, analogous to first passage percolation. When the percolation is critical the random geometric structure obtained is rather different.

We refer to the above random graph $G$ as CCCP (Contracting Clusters of Critical Percolation). For example (with Ori Gurel-Gurevich and Gady Kozma) we have: when $d=2$, the CCCP has exponential volume growth a.s. When $d>6$ a.s. the CCCP has double-exponential volume growth.

\section{Random planar metric}

Above we reviewed random perturbations of the Euclidean plane. How to define and model a genuine random planar metric?

\subsection{Local convergence}

Plane topology Angel and Schramm [3, 4] constructed the uniform infinite planar triangulation (UIPT), a rooted infinite random triangulation which is the limit (in the sense of [13]) of finite random triangulations (the uniform measure on all nonisomorphic triangulations of the sphere of size $n$ ), a model that was studied extensively by many (see e.g. [41]). The UIPQ is a similar construction with quadrangulation. The UIPT/Q looks very different from random perturbations of the plane as in the Poisson-Voronoi triangulation and has a rather surprising geometry at first encounter, e.g. volume growth of balls in the UIPT is asymptotically $r^{4}$. The UIPQ is recurrent [34] and subdiffusive [9] for the simple random walk and in particular hyperfinite. A collection of graphs is hypefinite if for every $\epsilon>0$ there is some finite $k$ such that each graph $G$ in the collection can be broken into connected components of size at most $k$ such that each has a boundary of size at most $\epsilon$ of its size. What about a hyperbolic nonhyperfinite counterpart? 
Hyperbolic analog? Guth, Parlier and Young [35] studied pants decomposition of random closed surfaces obtained by randomly gluing $N$ Euclidean triangles (with unit side length) together. They gave bounds on the size of pants decomposition of random compact surfaces with no genus restriction as a function of $N$. Their work indicates that the injectivity radius around a typical point is growing to infinity. Gamburd and Makover [30] showed that as $N$ grows the genus will converge to $N / 4$ and by Euler's characteristic the average degree will grow to infinity. What about a local limit of random finite triangulation/quadrangulation with genus growing linearly in the number of quadrangulation.

In the quadrangulation bijective techniques help a lot see [51]. In particular, Chassaing and Durhuus constructed the UIPQ from a random infinite labeled tree, followed by another construction in [21] from a labeled critical geometric Galton-Watson tree conditioned to survive. With Nicolas Curien we propose a model of infinite random quadrangulation constructed similarly from a labeled supercritical Galton-Watson tree. We conjecture that such a stochastic hyperbolic infinite quadrangulation (shiq) describes the limit of random finite quadrangulations with genus growing linearly in the number of quadrangulation. The Shiq is not hyperfinite and the simple random walk on the Shiq has positive speed almost surely.

Kaibel and Ziegler [37] survey a model of random lattice triangulations. They proved the existence of local limit and studying its properties, such as volume growth, seems interesting.

\subsection{Global convergence}

Scaling limits of random triangulations were also studied, see Le Gall [43] and Miermont [45] advancing over [20], who proved that the random triangulations scaled Gromov-Hausdorff converge to a random compact metric space of dimension 4. This limiting surface called the Brownian map can be seen as the two-dimensional sphere equipped with a random metric which induces the usual topology but makes it a fractal space of Hausdorff dimension 4. It is of interest to obtain quantitative estimates on the rate of convergence as in the Hungarian coupling of random walks and Brownian motion [40]. Also this theory is believed to be connected to 2D quantum gravity and conformal invariance via the following construction:

Conformal invariance Let $T_{n}$ be is a uniform triangulation of the sphere with $n$ faces. It is possible to get a "canonical" drawing of $T_{n}$ on the sphere by conformal tools. E.g. if $T_{n}$ has no loops or multiple edges, we can use the well-known circle packing theorem (see Wikipedia, [27]):

Theorem 8.1. If $T$ is a finite triangulation without loops or multiple edges then there exists a circle packing $P=\left(P_{c}\right)_{c \in C}$ in the sphere $\mathbb{S}_{2}$ such that the contact graph of $P$ is $T$. This packing is unique up to Möbius transformations.

The circle packing enables us to take a "nice" representation of a triangulation, nevertheless the non-uniqueness is somehow disturbing because to fix a representation we can, for example, fix the 
images of three uniformly chosen vertices of $T_{n}$. Once this is done, we form the atomic measure $\mu_{T_{n}}$ formed by the Dirac's at centers of the circles of the packing of $T_{n}$ renormalized to have mass one. This constitutes a canonical discrete conformal random probability on the sphere. By standard arguments there exist weak limits $\mu_{\infty}$ of $\mu_{T_{n}}$. Here are some tougher questions:

\section{Questions}

1. (Schramm [Talk about QG]) Determine coarse properties (invariant under $\mathrm{SO}_{3}(\mathbb{R})$ ) of $\mu_{\infty}$, e.g. what is the dimension of the support? Start with showing singularity.

2. Uniqueness (in law) of $\mu_{\infty}$ ? In particular can we describe $\mu_{\infty}$ in terms of Gaussian Free Field $(\mathrm{GFF}) ?$

Is it $\exp \left((8 / 3)^{1 / 2} G F F\right)$, does KPZ hold? See [25].

3. The random measure $\mu_{\infty}$ can come together with $\mathrm{d}_{\infty}$ a random distance on $\mathbb{S}_{2}$ (in the spirit of [42]). Can you describe links between $\mu_{\infty}$ and $\mathrm{d}_{\infty}$ ? Does one characterize the other? Is it a path metric space? See [31].

\subsection{Recursive subdivision}

Important properties of the UIPT holds for a larger family of planar graphs. Start with a finite directed graph and two marked vertices, one with one edge going out and one with one edge coming in and no other edges. Recursively replace each edge with a copy of the graph with the marked vertices mapped to the two vertices defining the edge. Extension of this scheme by recursively replacing fixed subgraphs results in infinite graphs admitting the doubling property: There is $C<\infty$, such that for any $r$, the size of any ball of radius $2 r$ in $G$ is bounded by $C$ times the size of a ball of radius $r$. For example the graph of the Sierpinski gasket satisfies this property.

By recursive subdivision one can construct planar graphs that have polynomial growth with arbitrarily large exponent. Still all these graphs are small in the following two senses. First, local limits of sequences of bounded degree planar graphs obtained by taking consecutive subdivisions are recurrent [13]. Second, in [12] the following is proved.

Theorem 8.2. Let $G$ be a planar graph such that the volume function of $G$ satisfies the doubling property. Then for every vertex $v$ of $G$ and radius $r$, there is a connected subset $\Omega$ such that $B(v, r) \subset$ $\Omega, \Omega \subset B(v, 6 r)$ and the size of the boundary of $\Omega$ is at most of order $r$.

Try to imagine the geometry of a planar recursive subdivision graph, when the volume growth is faster than quadratic. The facts above suggest heuristically that volume is generated by large fractal mushrooms like folds, and that the complements of balls have many connected components.

In particular we conjecture that the simple random walk spends a long time in such traps and hence is subdiffusive (that is, $\operatorname{dist}\left(o, X_{n}\right) \asymp n^{\alpha}$ where $X_{n}$ denotes the simple random walk starting at 
$o$ and $\alpha<1 / 2$ ). Is the critical probability of site percolation on any planar triangulation of uniform growth faster than quadratic $1 / 2$ ?

Here is a sketch of the proof of theorem 8.2. Let $v$ be any vertex of $\Gamma$. Consider the balls $B(v, n), B(v, 3 n)$. Let $N$ be an $n$-net of the boundary $\partial B(v, 3 n)$. For each vertex $w$ of $N$ consider $B(w, n / 2)$. Note that all such balls are disjoint since $N$ is an $n$-net. Also all these balls are contained in $B(v, 4 n)$. So, by the doubling property, we can have only boundedly many such balls, that is $|N| \leq \beta$, where $\beta$ does not depend on $n$. Consider now the balls $B(w, 2 n)$ for all $w \in N$. $\partial B(v, 3 n)$ is contained in the union of these balls. Construct a closed curve that 'blocks' $v$ from infinity as follows: if $w_{1}, w_{2} \in N$ are such that $d\left(w_{1}, w_{2}\right) \leq 2 n$ then we join them by a geodesic. So replace $\partial B(v, 3 n)$ by the 'polygonal line' that we define using vertices in $N$. This 'polygonal line' blocks $v$ from infinity and has length at most $2 n \beta$. There are some technical issues to take care of, for example $\partial B(v, 3 n)$ might not be connected (and could even have 'large gaps') and the geodesic segments have to be chosen carefully.

\section{Random subdivision}

There is growing interest in establishing a rigorous theory of two dimensional continuum quantum gravity. Heuristically, quantum gravity is a metric chosen on the sphere uniformly among all possible metrics. Although there are successful discrete mathematical quantum gravity models, we do not yet have a satisfactory continuum definition of a planar random length metric space (rather than random measure). One possible toy model is to start with a unit square, divide it into four squares and then recursively at each stage pick a square uniformly at random from the current squares (ignoring their sizes) and divide it to four squares and so on. Look at the minimal number of squares needed in order to connect the bottom left and top right corner with a connected set of squares. We conjecture that there is a deterministic scaling function, such that after dividing the random minimal number of squares needed after $n$ subdivisions by it, the result is a non degenerate random variable. Establishing the conjecture will provide a random planar length metric space. Does the shortest geodesic stabilizes as we further divide?

Since the conjecture seems hard, we start by studying the simplest recursive constructions after trees. As you will see below even here we mostly have questions and conjectures. The section is based on an ongoing project with Nicolas Curien.

\section{1 (Fixed) Hierarchical graphs}

Let us introduce the graphs we will work with. We start with a pattern, that is a finite connected graph $G_{1}$ with two distinguished point "source" and "sink" and such that the edges are oriented from source to sink. Inductively, the graph $G_{n}$ is constructed from $G_{n-1}$ by replacing each of its 
(oriented) edge by a copy of $G_{1}$ (source and sink respectively on the origin and target of the edge), see Fig. below.

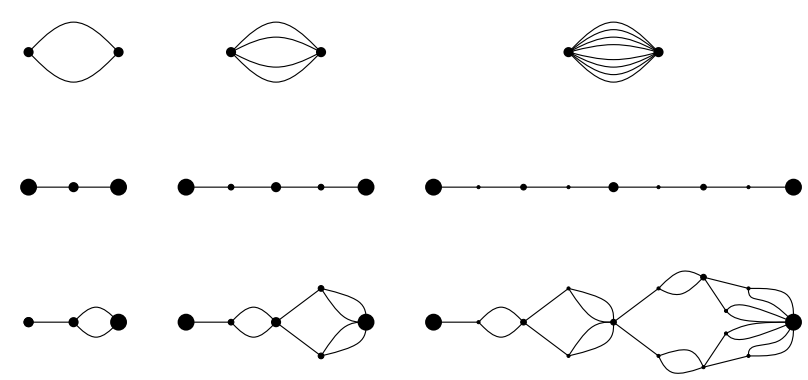

Figure 1: A few examples of hierarchical graphs

\subsection{Distance}

Fix a pattern $G_{1}$ and consider the sequence of hierarchical graphs $G_{1}, G_{2}, \ldots$ constructed as above. We endow these graphs with a random distance (or first passage percolation) model on them: assign a positive weight (e.g. uniform over $[0,1]$ ) independently for each edge of $G_{n}$. Recall that $G_{n}$ has two distinguished points "source" and "sink" and put

$$
D_{n}:=\text { Weight of a minimal path linking source and sink in } G_{n} \text {. }
$$

Obviously the $D_{n}$ 's satisfy a recursive distributional equation that is closely related to the initial pattern, e.g. for the three examples presented above we have for all $n \geq 2$

$$
\begin{aligned}
& D_{n} \stackrel{(d)}{=} \min \left(D_{n-1}, D_{n-1}^{\prime}\right) \\
& D_{n} \stackrel{(d)}{=} D_{n-1}+D_{n-1}^{\prime} \\
& D_{n} \stackrel{(d)}{=} D_{n-1}+\min \left(D_{n-1}^{\prime}, D_{n-1}^{\prime \prime}\right) \text {, }
\end{aligned}
$$

where $D_{n-1}, D_{n-1}^{\prime}, D_{n-1}^{\prime \prime}$ are independent copies of $D_{n-1}$. The first two equations are straightforward to analyze but the last one is thorny because the recursive distributional equation combines + (adding an edge in series) and min (presence of cycles). We focus on the last case. Let us consider a (wellknown) simplified model for the sake of comparison:

COMPARISON WITH BRANCHING RANDOM WALK. Consider $T_{n}$ the full binary tree starting with an edge up to level $n$ where each edge has been given an independent weight as above. In this case, the weight of the shortest path $M_{n}$ up to level $n$ satisfies

$$
M_{n}=\xi+\min \left(M_{n-1}, M_{n-1}^{\prime}\right)
$$


where $\xi$ denotes the law of the weights on the edges. In this model (first passage percolation on a tree) we know that $M_{n} \approx \gamma_{b r w} n$ with $\gamma_{b r w}$ explicit in terms of $\xi$ as well as the lower order terms. This is due to the fact that the geometry of the tree does not constrain the model too much and in that case $M_{n}$ is nearly obtained by considering all paths as independent. Also, a fairly simple argument due to Dekking and Host [23] shows that $M_{n}$ is strongly concentrated (order $O(1)$ ) around its mean. Let us sketch it. Provided that $\xi$ is bounded we can write

$$
M_{n} \leq C+\min \left(M_{n-1}, M_{n-1}^{\prime}\right) .
$$

Assume now that $M_{n-1}$ is not concentrated around its mean, the key is to notice that in this case we have

$$
E\left[\min \left(M_{n-1}, M_{n-1}^{\prime}\right)\right] \text { sensibly less than } E\left[M_{n-1}\right] \text {. }
$$

Taking expectation we deduce that $E\left[M_{n}\right]$ is noticeably less than $E\left[M_{n-1}\right]+C$ however this cannot be the case since $E\left[M_{n}\right] \geq E\left[M_{n-1}\right]$.

Coming back to (1). We will compare $D_{n}$ with $M_{2^{n}}$ (the $2^{n}$ comes from the fact that the height of the graph $G_{n}$ is $2^{n}$ compared to the height $n$ of $T_{n}$ ). Clearly we have $D_{n} \leq 2^{n}$ and one can also show by induction that $D_{n} \geq M_{2^{n}}$, indeed notice that

$$
M_{2^{n}} \leq M_{2^{n-1}}+\min \left(M_{2^{n-1}}^{\prime}, M_{2^{n-1}}^{\prime \prime}\right),
$$

and then use (1). Hence we have $\gamma_{b r w} 2^{n} \leq E\left[D_{n}\right] \leq 2^{n}$ and a simple monotonicity argument shows that if $\xi$ is non-degenerate then $\gamma_{r e c}:=\lim 2^{-n} E\left[D_{n}\right]$ exists and is in $\left[\gamma_{b r w}, 1\right)$. In view of these remarks we have the following.

Question 9.1. Compute $\gamma_{r e c}$ in terms of $\xi$ in particular show (if true) $\gamma_{r e c}>\gamma_{b r w}$.

We think that the convergence in mean of $D_{n}$ implies (thanks to (1)) its convergence in probability. However subtle questions about $D_{n}$ remain open.

Question 9.2. What is the concentration of $D_{n}$ around its mean? Lower order terms? More generally, ask the same questions as for the minimal position in a branching random walk.

For background on branching random walks see e.g. [53]

\subsection{External DLA}

In the hierarchical graph $G_{n}$ we launch particles one by one from the sink. The particle performs SRW and settles as soon as it hits a vertex adjacent to the source or previously settled particle. This is the standard model of External Diffusion Limited Aggregation on $G_{n}$. This process ends when a particle settles at the sink.

What is the proportion of $G_{n}$ that is covered? 
We denote by $P_{n}$ the number of particles launched before the end of the process. Using the recursive structure of the graph $G_{n}$ we can also write a recursive distributional equation for $P_{n}$ e.g. in the third case of Fig. 1 neglecting a few terms we have

$$
P_{n}=P_{n-1}+2 \min \left(P_{n-1}^{\prime}, P_{n-1}^{\prime \prime}\right)
$$

Compare with (1) (the presence of the factor 2 stems from the fact that the particles starting from the sink in $G_{n}$ are (roughly speaking) split into two equal groups of particles in the two branches of the initial $G_{1}$ ). Note that the number of edges in $G_{n}$ is $3^{n}$ so knowing whether $G_{n}$ is almost full of particles is the same as knowing whether $E\left[P_{n}\right]$ is sensibly less than $3 E\left[P_{n-1}\right]$ or not. Notice that if $P_{n-1}$ is not concentrated then $2 \min \left(P_{n-1}^{\prime}, P_{n-1}^{\prime \prime}\right)$ is say less than $(2-\varepsilon) E\left[P_{n-1}\right]$ thus $E\left[P_{n}\right]<(3-\varepsilon) D_{n}$. But if $P_{n-1}$ is concentrated we cannot say anything.

Question 9.3. What is $\lim n^{-1} \log \left(E\left[P_{n}\right]\right)$ ?

\subsubsection{The win-win situation}

Knowing whether the graph is full or not can be answered for a special type of recursive graph where "a shadowing effect"` takes place. Indeed, consider the sequence of graphs $G_{n}$ with initial pattern $\bullet \downarrow \cdot$, its fourth iteration is the figure below. In this case we can still write recursive distributional

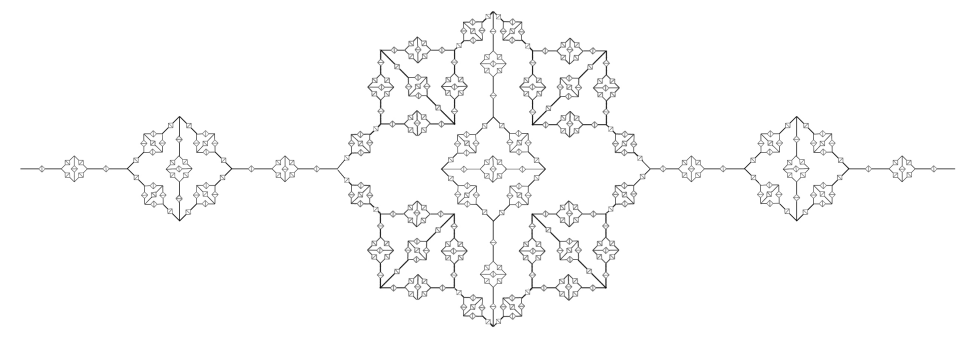

Figure 2: Naomi's fractal

equations for the $P_{n}$ but the heuristic argument goes as follows. Notice first that the volume of the graph grows like $7^{n}$ so we have to compare $E\left[P_{n}\right]$ with $7 E\left[P_{n-1}\right]$. If $P_{n-1}$ is not concentrated then $E\left[P_{n}\right]<(7-\varepsilon) E\left[P_{n-1}\right]$ as above and we are done. So assume that $P_{n-1}$ is concentrated. In the filling process of $G_{n}$ the (offspring of the) first branch in $G_{1}$ linked to the source will be filled first which takes a time $P_{n-1}$ and then the two branches adjacent to this one start to be filled. The key 
point is to notice that since $P_{n-1}$ is concentrated, these two branches will be totally full at roughly the same time. In which case the branch of the "middle" will receive no particles due to a shadowing effect of the last two branches. Finally in this case we expect $E\left[P_{n}\right] \approx 6 E\left[P_{n-1}\right]$. We thus see that in all situations $E\left[P_{n}\right]<(7-\varepsilon) E\left[P_{n-1}\right]$ and the following result essentially follows.

Proposition 9.4 (with Nicolas Curien). We have $\lim \sup n^{-1} \log \left(E\left[P_{n}\right]\right)<7$ and hence the graph $G_{n}$ is not totally filled during the EDLA process, more precisely the aggregate covers a fractal portion of it.

It will be nice to show the same for other fractals, starting with Sierpinski gasket.

\subsection{Random hierarchical graphs}

In this section, the graph we build are themselves random but still based on a hierarchical procedure. Let us describe one possible model. We start with a pattern $G_{1}$. To get $G_{n}$ from $G_{n-1}$ we first pick one edge of $G_{n-1}$ uniformly at random and replace it by a copy of the initial pattern $G_{1}$. See Fig. ref below for an example. Using connection with branching processes, Thomas Duquesne (private

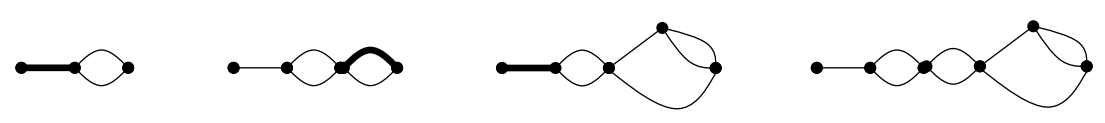

Figure 3: Construction with the third pattern of Fig. 1

communication) has been able to compute exactly the expectations of the number of oriented paths going from left to right in $G_{n}$. We denote by $\mathcal{D}_{n}$ the distance between the two extremal points in $G_{n}$. Trivially $\mathcal{D}_{n} \leq n+1$. A fairly simple sub-additivity argument shows that in fact

$$
\frac{\log \left(E\left[\mathcal{D}_{n}\right]\right)}{\log (n)} \underset{n \rightarrow \infty}{\longrightarrow} \gamma \in[0,1]
$$

Ad-hoc calculations show that $\gamma \in(\varepsilon, 1 / 2-\varepsilon)$. But the true value of $\gamma$ remains mysterious. This model is intimately connected to a urn model : The volume of the graphs offspring of the three original edges form a standard Polya urn $^{1}$. So the limiting proportions of edges in these graphs $\left(\alpha_{1}, \alpha_{2}, \alpha_{3}\right)$ is distributed as a Dirichlet distribution of parameters $(1 / 2,1 / 2,1 / 2)$. Thus, loosely speaking, the recursive distributional equations satisfied by the $\mathcal{D}_{n}$ 's are the following

$$
\mathcal{D}_{n} \stackrel{(d)}{=} \mathcal{D}_{\alpha_{1} n}+\min \left(\mathcal{D}_{\alpha_{2} n}^{\prime}, \mathcal{D}_{\alpha_{3} n}^{\prime \prime}\right)
$$

where $\left(D_{n}\right),\left(D_{n}^{\prime}\right)$ and $\left(D_{n}^{\prime \prime}\right)$ are copies of the original process and independent of the $\left(\alpha_{1}, \alpha_{2}, \alpha_{3}\right)$. In this model, the non-concentration of $\mathcal{D}_{n}$ is granted so the interesting questions are the following.

\footnotetext{
${ }^{1}$ three balls of three colors initially, when a ball is picked it is replaced in the urn together with 2 balls of the same color
} 
Question 9.5. What is the value of $\gamma$ ? Can we rescale $\mathcal{D}_{n}$ to have convergence in distribution? (this is equivalent to the Gromov-Hausdorff convergence of the rescaled graphs).

Finally, we mention a last model in the same spirit. This is the series-parallel random graph introduced by Hambly and Jordan [36]. Fix a parameter $p \in[0,1]$. The construction goes as follows. We start with a single edge. Then inductively at each stage, all the edges of the graph are replaced by two edges in series with probability $p$ or two edges in parallel with probability $1-p$. If $\Delta_{n}$ is the distance between the two extremal points in this graph then the recursive distributional equations are now

$$
\Delta_{n} \stackrel{(d)}{=}\left\{\begin{array}{lc}
\text { with proba } p, & \Delta_{n-1}+\Delta_{n-1}^{\prime} \\
\text { with proba } 1-p, & \min \left(\Delta_{n-1}, \Delta_{n-1}^{\prime}\right) .
\end{array}\right.
$$

It is easy to see that when $p<1 / 2$ then $\Delta_{n}$ remains bounded. However, when $p>1 / 2$ this distance grows exponentially with $n$ and by a subadditivity argument we get

$$
E\left[\Delta_{n}\right] \approx e^{n \delta(p)+o(n)} .
$$

Question 9.6. What is the shape of $p \in[1 / 2,1] \mapsto \delta(p)$. In particular, do we have $\delta(1 / 2)=0$ ?

Acknowledgements: Thanks to Nicolas Curien for substantial help with the writing and Naomi Benjamini for the drawing.

\section{References}

[1] D. Aldous, Scale-invariant random spatial networks, arXiv:1204.0817

[2] D. Aldous and R. Lyons, Processes on unimodular random networks, Electron. J. Prob. paper 54, pages 1454-ñ1508 (2007).

[3] O. Angel, Growth and percolation on the uniform infinite planar triangulation, Geometric And Functional Analysis 13 935-974 (2003).

[4] O. Angel and O. Schramm, Uniform infinite planar triangulations, Comm. Math. Phys. 241 , 191-213 (2003).

[5] L. Babai and M. Szegedy, Local expansion of symmetrical graphs, Combinatorics, Probability and Computing (1992), 1: 1-11

[6] R. Basu and A. Sly, Lipschitz embeddings of random sequences, arXiv:1204.2931

[7] I. Benjamini and N. Curien, On limits of graphs sphere packed in Euclidean space and applications. European J. Comb. 32 975ñ-984, (2011). 
[8] I. Benjamini and N. Curien, Ergodic theory on stationary random graphs, Electron. J. Probab. 1720 pp. (2012).

[9] I. Benjamini and N. Curien, Simple random walk on the uniform infinite planar quadrangulation: Subdiffusivity via pioneer points, (2012) GAFA to appear.

[10] I. Benjamini, H. Finucane and R. Tessera, On the scaling limit of finite vertex transitive graphs with large diameter, arXiv:1203.5624

[11] I. Benjamini, G. Kalai and O. Schramm, First passage percolation has sublinear distance variance, Ann. of Prob. 31 1970-1978 (2003).

[12] I. Benjamini and P. Papasoglu, Growth and isoperimetric profile of planar graphs, Proc. Amer. Math. Soc. 139 (2011), no. 11, 4105-4111.

[13] I. Benjamini and O. Schramm, Recurrence of Distributional Limits of Finite Planar Graphs, Electron. J. Probab. 613 pp. (2001).

[14] I. Benjamini, O. Schramm and A. Timar, On the separation profile of infinite graphs, Group, Geometry and Dynamics. 6 pp. 639ñ-658 (2012).

[15] M. Biskup, On the scaling of the chemical distance in long-range percolation models, Ann. Prob. 32 2938-2977 (2004).

[16] B. Bowditch, A short proof that a subquadratic isoperimetric inequality implies a linear one, Michigan Math. J. 42 (1995) 103-107

[17] E. Breuillard, B. Green and T. Tao, The structure of approximate groups, arXiv:1110.5008

[18] D. Burago, Y. Burago, and S. Ivanov, A course in metric geometry, Graduate Studies in Mathematics. American Mathematical Society, Providence, RI, (2001).

[19] D. Burago and S. Ivanov, Uniform approximation of metrics by graphs, arXiv:1210.2435

[20] P. Chassaing and G. Schaeffer, Random planar lattices and integrated superBrownian excursion, Prob. Theor. and Rel. Fields 128, 161-212 (2004).

[21] N. Curien, L. Ménard and G. Miermont, A view from infinity of the uniform infinite planar quadrangulation. http://arxiv.org/abs/1201.1052

[22] M Deijfen, O Häggström, The pleasures and pains of studying the two-type Richardson model, Analysis and Stochastics of Growth Processes and interface models, 39-54 (2008).

[23] F. Dekking and B. Host, Limit distributions for minimal displacement of branching random walks, Probab. Theory Relat. Fields 90, 403-426 (1991). 
[24] J. Ding and A. Sly, Distances in critical long range percolation, arXiv:1303.3995

[25] B. Duplantier and S. Sheffield, Liouville quantum gravity and KPZ, Invensiones Mathematicae 185, 333-393 (2011).

[26] O. Häggström and R. Pemantle, First passage percolation and a model for competing spatial growth, Jour. Appl. Proba. 35 683-692 (1998)

[27] Z-X. He and O. Schramm, Hyperbolic and parabolic packings, Jour. Discrete and Computational Geometry 14 123-149, (1995).

[28] C. Hoffman, Geodesics in first passage percolation, Ann. Appl. Prob. 18,1944-1969 (2008).

[29] D. Howard and C. Newman, Euclidean models of first-passage percolation, Probab. Theory Related Fields 108, 153-170 (1997).

[30] A. Gamburd and E. Makover, On the genus of a random Riemann surface, Contemp. Math.. 311 (2002), 133-ñ140.

[31] C. Garban, R. Rhodes and V. Vargas, Liouville Brownian motion, http://arxiv.org/abs/1301.2876

[32] T. Gelander, A metric version of the Jordan-Turing theorem, arXiv:1205.6553

[33] G. Grimmett and H. Kesten, Percolation since Saint-Flour, arXiv:1207.0373

[34] O. Gurel-Gurevich and A. Nachmias, Recurrence of planar graph limits, Annals of Math. To appear (2012).

[35] L. Guth, H. Parlier and R. Young, Pants decompositions of random surfaces, arXiv:1011.0612

[36] B. Hambly and J. Jordan, A random hierarchical lattice: the series-parallel graph and its properties, Adv. Appl. Prob. 36, 824-838 (2004).

[37] V. Kaibel and G. Ziegler, Counting lattice triangulations, London Math. Society Lecture Notes Series, 307, 277-307 (2003).

[38] H. Kesten, The critical probability of bond percolation on the square lattice equals $1 / 2$, Comm. Math. Phys. 74 (1980), no. 1, 41-59.

[39] H. Kesten, Aspects of first passage percolation, Lecture Notes in Math,Springer (1986)

[40] J. Komlos, P. Major and G. Tusnady, An approximation of partial sums of independent RV'-s, and the sample DF. I, Prob. Theor. and Rel. Fields. 32, (1975), 111-131. 
[41] M. Krikun, A uniformly distributed infinite planar triangulation and a related branching process, Zap. Nauchn. Sem. S.-Peterburg. Otdel. Mat. Inst. Steklov. (POMI), 307(Teor. Predst. Din. Sist. Komb. i Algoritm. Metody. 10):141-174, 282-283 (2004).

[42] J.F. Le Gall, The topological structure of scaling limits of large planar maps, Invent. Math., 169(3):621-670 (2007).

[43] J.F. Le Gall, Uniqueness and universality of the Brownian map, arXiv:1105.4842

[44] L. Lovász, Large networks, graph homomorphisms and graph limits, Amer. Math. Soc. In preparation (2012).

[45] G. Miermont, The Brownian map is the scaling limit of uniform random plane quadrangulations, arXiv:1104.1606

[46] G. Miller, S. Teng, W. Thurston and S. Vavasis, Geometric separators for finite-element meshes, SIAM J. Sci. Comput. 19 (1998), no. 2, 364-386 (electronic).

[47] V. Nekrashevych and G. Pete, Scale-invariant groups, Groups, Geometry and Dynamics 5 (2011), 139-167.

[48] P. Pansu, Croissance des boules et des géodésiques fermées dans les nilvariétés, Ergodic Theory Dyn. Syst. 3, 415-445 (1983).

[49] P. Pansu, Large scale conformal maps, Preprint (2012).

[50] C. Radin, The Pinwheel tilings of the plane, Annals of Mathematics 139 (3): pp.661-702 (1994).

[51] G. Schaeffer, Conjugaison d'arbres et cartes combinatoires aléatoires, phd thesis, (1998).

[52] N. Varopoulos, L. Saloff-Coste and T. Coulhon, Analysis and geometry on groups, Cambridge University Press (1993).

[53] O. Zeitouni, Branching random walks and Gaussian fields Notes for Lectures, http://www.wisdom.weizmann.ac.il/ zeitouni/pdf/notesBRW.pdf 